\title{
FASNating Targets of Metformin in Breast Cancer Stem-Like Cells
}

\author{
Elizabeth A. Wellberg • Steven M. Anderson
}

Received: 24 July 2014 / Accepted: 30 July 2014 /Published online: 30 August 2014

(C) Springer Science+Business Media New York 2014

\section{Diabetes, Breast Cancer, and Metformin}

Emerging data suggest that metformin treatment is associated with reduced breast cancer incidence and mortality for individuals with type II diabetes [7, 6, 17]. For years, many studies have been done to investigate the possible direct and indirect effects of metformin on cancers of various origins. In diabetic patients, metformin improves metabolic function by promoting muscle glucose uptake and lowering hepatic gluconeogenesis $[14,15,36]$. In cultured cancer and normal cells, metformin has pleiotropic effects, including decreasing mitochondrial electron transport complex I activity, increasing the cellular AMP to ATP ratio, and activating the AMPK signaling pathway. The consequence of this is reduced protein translation, DNA synthesis, and cell proliferation. In isolated cancer stem-like cells (CSC), metformin's targets range from inflammation [13], to micro-RNAs [1], to the synthesis of nucleotide triphosphates [16]. The diversity of responses elicited by metformin from different tissues and from the specific cell types within those tissues suggests that metformin may be a valuable therapeutic for the treatment of a variety of cancers at many stages of tumor progression. Indeed, scores of clinical trials aiming to investigate the therapeutic effects of metformin for patients with cancer are either planned or ongoing. For breast cancer, large trials are open for patients with early-stage disease (NCT01101438) and metastatic disease (NCT01310231). The results of these trials are highly anticipated and will likely answer many lingering questions about the efficacy of metformin against cancer in nondiabetic patients. Short-term trials have also been conducted to evaluate the effects of metformin on breast tumor cell proliferation (NCT00897884) and tumor cell metabolism (NCT01266486).

E. A. Wellberg $\cdot$ S. M. Anderson $(\bowtie)$

Department of Pathology, University of Colorado School of

Medicine, University of Colorado Anschutz Medical Campus, Mail

Stop 8104, 12801 East 17th Avenue, Aurora, CO 80045, USA

e-mail: Steve.Anderson@ucdenver.edu
In this issue, Wahdan-Alaswad et al. demonstrate that in ER/PR/HER2-negative (triple negative (TN)) breast cancer cells metformin treatment targets fatty acid synthase (FASN) through a miR193b-dependent mechanism. Specifically, in TN breast cancer cells, metformin upregulated miR193b, which directly targeted the FASN mRNA resulting in decreased FASN protein levels. This was associated with reduced proliferation and increased apoptosis. Gene expression profiling also revealed changes in other metabolic enzymes, including members of the cholesterol biosynthesis pathway, suggesting that metformin may impact cancer cell lipid metabolism networks at multiple nodes. One limitation of this study is that the levels of cellular fatty acids were not quantified. Presumably, the amounts of de novo synthesized fatty acids correlate with the levels of FASN protein. In cancer cells, FASN expression is regulated by the HER2/Neu and PI3K signaling pathways, steroid hormones, and genomic amplification [20,34, 37, 42]; however, the spectrum of fatty acid products and the kinetics of the FASN reaction can be altered by effector proteins [21,32]. Interestingly, the effects of metformin on miR193b and FASN were only observed when cells were grown in media containing physiologic glucose levels $(5 \mathrm{mM})$ compared to high glucose $(17 \mathrm{mM})$. The metformin-mediated increase in miR193b inhibited mammosphere formation and also reduced the population of $\mathrm{CSC}\left(\mathrm{CD} 24^{\text {low }} / \mathrm{CD} 44^{\mathrm{hi}} / \mathrm{ALDH}^{+}\right)$; all of these effects were blocked by antagomirs of miR193b. Inhibition of FASN using C75 or cerulenin also reduced mammosphere formation, suggesting that CSC, particularly of the TN subtype, are critically dependent on FASN activity.

\section{Cell Type-Specific Metabolic Activity}

The FASN enzyme is frequently elevated in solid tumors from a variety of tissues compared to surrounding normal cells, and this appears to occur early during tumorigenesis (reviewed in [19]). Studies have shown that the majority of fatty acids in 
cancer cells are derived from de novo synthesis regardless of the availability of preformed extracellular lipids derived from the diet or adipose stores [27,33]. This observation suggests that the de novo synthesized fatty acids meet a specific need during tumorigenesis or tumor progression, which cannot be met by dietary lipids. Increased de novo fatty acid synthesis provides several advantages to tumors. Cancer cells are rapidly dividing and have a heightened requirement for membrane phospholipid precursors. In addition, certain fatty acids can regulate intracellular signaling pathways either by activating signaling molecules or through posttranslational modification of pro-tumorigenic kinases, such as Ras and Src [22, $30,31]$. Because of the apparent dependence of early tumor growth on the FASN enzyme, many studies have focused on it and other members of the fatty acid synthesis pathway for cancer therapy.

Recent studies have provided insight into metabolic differences between stem cells and somatic cells, as well as CSC and non-stem cancer cells. Stem cells, including embryonic stem cells (ESC), hematopoietic stem cells (HSC), and induced pluripotent stem cells (iPSC), appear to rely on glycolysis rather than mitochondrial respiration for the production of ATP (reviewed in [29]). The metabolomes of iPSC and ESC are closely related to each other and are consistent with observations that inhibition of oxidative pathways sustains pluripotency [28, 41]. Specifically, iPSC and ESC have a decrease in the metabolites derived from mitochondrial respiration and an increase in glycolytic intermediates [28]. The metabolic activity of stem cells may be unique, depending on what differentiation pathway the cells adopt. For example, HSC display particular requirements for either glucose or glutamine, depending upon whether differentiating along the erythroid or myeloid pathways [26]. A recent study demonstrated that FASN activity is critical for maintaining the population of neural stem/progenitor cells [18], in a manner similar to that described by Wahdan-Alaswad et al. If de novo synthesized fatty acids are important in maintaining stem cells in an undifferentiated state, it is not surprising that these cells become glucose-dependent in order to generate the building blocks for fatty acids.

The metabolic profile of CSC in breast cancer remains controversial and understudied. One study used a proteomics approach to determine that breast CSC rely on glycolysis more than differentiated breast cancer cells [8], which is consistent with observations made in noncancer stem cells. A different group demonstrated elevated glucose consumption and ATP levels, reduced lactate production, and a greater reliance on mitochondrial respiration in breast CSC compared to differentiated cells, indicating the absence of the classic Warburg phenotype [38]. Stem cells present in primary glioblastoma were observed to be highly glucose-dependent and under conditions of low glucose availability, they upregulated GLUT3. Compared to GLUT1, the GLUT3 transporter has a higher affinity for glucose, and its increased expression would provide a competitive advantage for a cell in an environment with limited glucose. It is currently unknown precisely how circulating glucose impacts stem-like cell populations in breast cancer. Based on published studies, one could postulate that elevated glucose, such as that associated with type II diabetes, would support the expansion of breast CSC pools. On the contrary, it is unclear if breast CSC are uniquely able to survive and/or expand under conditions of limited glucose. Future studies will surely address the mechanisms through which peripheral metabolic disease alters CSC survival, proliferation, and differentiation. While the observations that some CSC are dependent on glucose have provided insight into their metabolism, it will be important to identify precisely how these cells use glucose (i.e., aerobic glycolysis versus mitochondrial respiration) in order to develop therapies that selectively eliminate this cell population. Finally, to develop metabolism-based therapies with specificity to CSC, it will be critical to define the precise metabolic differences that exist between them and noncancer stem cells.

\section{Breast Cancer Subtypes and Warburg Metabolism}

While it is a relatively rare form of breast cancer, the TN subtype presents unique challenges for both physicians and patients (reviewed in [11]). It is called "triple negative" because it lacks ER, PR, and HER2, making targeted therapy ineffective. Instead, many patients must take chemotherapy, which often has undesirable side effects. The observation that TN breast cancer cells are more sensitive to metformin than ER/PR/HER2-positive cells opens the door for the development of novel therapeutic strategies that may lead to a better quality of life for patients with TN disease. Wahdan-Alaswad and others $[25,39]$ have previously demonstrated that breast cancer cells are more sensitive to metformin when cultured in media containing physiologic $(5 \mathrm{mM})$ levels of glucose compared to media containing high glucose (10 to $25 \mathrm{mM}$ ). Their new study extends their previous work, which investigated the mechanisms through which metformin impacts breast cancer and how those mechanisms varied in the presence of different glucose concentrations across cell lines [39]. Regardless of breast cancer subtype, high glucose concentrations elevated cell proliferation and attenuated the effects of metformin on colony formation, cell cycle progression, apoptosis, and signaling pathway inhibition. TN breast tumors have been reported to take up more glucose compared to the ER/PR/ HER2-positive subtype [2], and TN breast cancer cells appeared to be more dependent on glycolysis than other subtypes $[9,24]$. Together, these studies suggest that TN breast cancers may be particularly sensitive to low glucose availability, potentially offering more effective treatment strategies for patients afflicted with this aggressive disease. 
The elevated dependence of cancer on glucose has been rigorously studied since it was first observed by Otto Warburg [40]. Initially, it was thought that cancer cell mitochondria were dysfunctional, as high glucose consumption and lactate production occurred in the presence of oxygen (aerobic glycolysis). Now, it appears that, in many cases, mitochondrial ATP production in cancer cells is not impaired, and the excess glucose is largely used for tangential pathways that produce nucleotides, reducing intermediates, amino acids, and fatty acid precursors (reviewed in [35]). By increasing glucose consumption, cancer cells are able to meet both their energetic needs and their need for molecular building blocks during the processes of transformation and tumor progression. Because the use of glucose is highly leveraged to produce so many different molecules, it is not surprising that metformin, by targeting mitochondrial respiration and ATP production, is able to compromise the growth of cells displaying the Warburg phenotype.

\section{Closing Remarks}

The article by Wahdan-Alaswad et al. demonstrates a novel link between metformin-mediated changes in micro-RNAs and cancer cell metabolic pathways, adding another potential mechanism of metformin action to the growing list. It also suggests several areas of future exploration. Data from the Thor and Richer laboratories and from others clearly demonstrate that careful consideration must be given to cell culture conditions when investigating mechanisms of metformin action in cancer [4, 25, 39,]. Cultured cancer cells are often propagated in media containing extraordinarily high levels of glucose, which far surpass physiological levels even in cases of uncontrolled diabetes. As postulated in a recent review by Dowling et al. [10], tumors developing in individuals with metabolic disease may have become dependent on elevated glucose and may be more sensitive to glucoselowering therapies than those tumors in nondiabetic individuals. This can be likened to acutely exposing cancer cells that are routinely cultured in high glucose to limited glucose, potentially leading to a stress response. Lowering the available glucose in culture may result in a unique sensitivity to drugs that target cellular metabolism.

The cancer-killing effects of metformin have been postulated to involve direct and indirect mechanisms (reviewed in [10]). Metformin improves peripheral metabolic function, which results in decreased circulating insulin and glucose and likely contributes to the beneficial effects of metformin observed in diabetic patients with cancer. By directly targeting cancer cells to activate the AMPK signaling cascade, metformin treatment leads to cellular changes that are consistent with an energy stress response. In addition to conserving energy by ceasing ATP-consuming pathways, cells increase glucose uptake to restore energy balance. Metformin was reported to stimulate glucose uptake in colon cancer cells in vitro [12, 23], yet this was associated with decreased cell proliferation [12]. When cells were grown as tumors in vivo, the effects of metformin on glucose uptake were mixed. In one study, metformin treatment increased tumor glucose uptake [12]. Another study reported a metformin-mediated reduction in the copious glucose uptake observed in tumors from mice that were fed a high fat diet [23]. These studies highlight a potential limitation of metformin therapy and indicate that further investigation is warranted into the effects of metformin on cancer cell glucose uptake in vivo.

The impact of metformin on cancer cell metabolism suggests a scenario where metformin treatment, by inhibiting mitochondrial function, would force cells to increase their dependence on other pathways for ATP production, such as glycolysis, autophagy, or oxidation of dietary fats. The greater sensitivity of breast cancer cells to the anticancer effects of metformin in low glucose supports this model and reveals numerous avenues for investigation into combination therapies for cancers of various origins. Indeed, the administration of metformin in combination with 2-deoxyglucose was more effective at killing gastric and esophageal cancer cells, as well as prostate cancer cells, than either compound alone [3, 5]. Likewise, colon cancer cells lacking p53 were shown to be exquisitely sensitive to metformin and unable to convert to a metabolic program that was dependent on glycolysis and autophagy, while cells with an intact p53 network increased their dependence on both pathways for survival when mitochondrial metabolism was impaired [4]. Finally, the differences in cellular metabolism between stem cells and differentiated cells from cancerous and noncancerous tissues may define a critical parameter in the effectiveness of anticancer therapy.

Acknowledgments This work was supported by a grant from the Public Health Service PO1-HD38129 awarded to SMA.

Conflict of Interest The authors declare that they have no conflicts of interest.

\section{References}

1. Bao B, Wang Z, Ali S, Ahmad A, Azmi AS, Sarkar SH, Banerjee S et al (2012) Metformin inhibits cell proliferation, migration and invasion by attenuating CSC function mediated by deregulating miRNAs in pancreatic cancer cells. Cancer Prev Res (Phila) 5(3): 355-364. doi:10.1158/1940-6207.CAPR-11-0299

2. Basu S, Chen W, Tchou J, Mavi A, Cermik T, Czerniecki B, Schnall M, Alavi A (2008) Comparison of triple-negative and estrogen receptor-positive/progesterone receptor-positive/HER2-negative breast carcinoma using quantitative fluorine-18 fluorodeoxyglucose/positron emission tomography imaging 
parameters: a potentially useful method for disease characterization. Cancer 112(5):995-1000. doi:10.1002/cncr.23226

3. Ben Sahra I, Laurent K, Giuliano S, Larbret F, Ponzio G, Gounon P, Le Marchand-Brustel $Y$ et al (2010) Targeting cancer cell metabolism: the combination of metformin and 2-deoxyglucose induces p53-dependent apoptosis in prostate cancer cells. Cancer Res 70(6): 2465-2475. doi:10.1158/0008-5472.CAN-09-2782

4. Buzzai M, Jones RG, Amaravadi RK, Lum JJ, DeBerardinis RJ, Zhao F, Viollet B, Thompson CB (2007) Systemic treatment with the antidiabetic drug metformin selectively impairs p53-deficient tumor cell growth. Cancer Res 67(14):6745-6752. doi:10.1158/ 0008-5472.CAN-06-4447

5. Cheong JH, Park ES, Liang J, Dennison JB, Tsavachidou D, NguyenCharles C, Wa Cheng K et al (2011) Dual inhibition of tumor energy pathway by 2-deoxyglucose and metformin is effective against a broad spectrum of preclinical cancer models. Mol Cancer Ther 10(12):2350-2362. doi:10.1158/1535-7163.MCT-11-0497

6. Chlebowski RR, Aragaki AK, McTiernan A (2013) Reply to S Gandini et al. J Clin Oncol 31(7):974-975

7. Chlebowski RT, McTiernan A, Wactawski-Wende J, Manson JE, Aragaki AK, Rohan T, Ipp E et al (2012) Diabetes, metformin, and breast cancer in postmenopausal women. J Clin Oncol 30(23):2844 2852. doi:10.1200/JCO.2011.39.7505

8. Ciavardelli D, Rossi C, Barcaroli D, Volpe S, Consalvo A, Zucchelli M, De Cola A et al (2014) Breast cancer stem cells rely on fermentative glycolysis and are sensitive to 2-deoxyglucose treatment. Cell Death Dis 5:e1336. doi:10.1038/cddis.2014.285

9. Dennison JB, Molina JR, Mitra S, Gonzalez-Angulo AM, Balko JM, Kuba MG, Sanders ME et al (2013) Lactate dehydrogenase B: a metabolic marker of response to neoadjuvant chemotherapy in breast cancer. Clin Cancer Res 19(13):3703-3713. doi:10.1158/1078-0432.CCR-130623

10. Dowling RJ, Niraula S, Stambolic V, Goodwin PJ (2012) Metformin in cancer: translational challenges. J Mol Endocrinol 48(3):R31-43. doi:10.1530/JME-12-0007

11. Engebraaten O, Vollan HK, Borresen-Dale AL (2013) Triplenegative breast cancer and the need for new therapeutic targets. Am J Pathol 183(4):1064-1074. doi:10.1016/j.ajpath.2013.05.033

12. Habibollahi P, van den Berg NS, Kuruppu D, Loda M, Mahmood U (2013) Metformin - an adjunct antineoplastic therapy — divergently modulates tumor metabolism and proliferation, interfering with early response prediction by 18 F-FDG PET imaging. J Nucl Med 54(2): 252-258. doi:10.2967/jnumed.112.107011

13. Hirsch HA, Iliopoulos D, Struhl K (2013) Metformin inhibits the inflammatory response associated with cellular transformation and cancer stem cell growth. Proc Natl Acad Sci U S A 110(3):972-977. doi:10.1073/pnas. 1221055110

14. Hundal HS, Ramlal T, Reyes R, Leiter LA, Klip A (1992) Cellular mechanism of metformin action involves glucose transporter translocation from an intracellular pool to the plasma membrane in L6 muscle cells. Endocrinology 131(3):1165-1173. doi:10.1210/endo. 131.3.1505458

15. Hundal RS, Krssak M, Dufour S, Laurent D, Lebon V, Chandramouli V, Inzucchi SE et al (2000) Mechanism by which metformin reduces glucose production in type 2 diabetes. Diabetes 49(12):2063-2069

16. Janzer A, German NJ, Gonzalez-Herrera KN, Asara JM, Haigis MC, Struhl K (2014) Metformin and phenformin deplete tricarboxylic acid cycle and glycolytic intermediates during cell transformation and NTPs in cancer stem cells. Proc Natl Acad Sci U S A. doi:10. 1073/pnas.1409844111

17. Jiralerspong S, Palla SL, Giordano SH, Meric-Bernstam F, Liedtke C, Barnett CM, Hsu L, Hung MC, Hortobagyi GN, Gonzalez-Angulo AM (2009) Metformin and pathologic complete responses to neoadjuvant chemotherapy in diabetic patients with breast cancer. J Clin Oncol 27(20):3297-3302. doi:10.1200/JCO.2009.19.6410
18. Knobloch M, Braun SM, Zurkirchen L, von Schoultz C, Zamboni N, Arauzo-Bravo MJ, Kovacs WJ et al (2013) Metabolic control of adult neural stem cell activity by Fasn-dependent lipogenesis. Nature 493(7431):226-230. doi:10.1038/nature11689

19. Kuhajda FP (2006) Fatty acid synthase and cancer: new application of an old pathway. Cancer Res 66(12):5977-5980. doi:10.1158/ 0008-5472.CAN-05-4673

20. Kumar-Sinha C, Ignatoski KW, Lippman ME, Ethier SP, Chinnaiyan AM (2003) Transcriptome analysis of HER2 reveals a molecular connection to fatty acid synthesis. Cancer Res 63(1):132-139

21. Libertini LJ, Smith S (1978) Purification and properties of a thioesterase from lactating rat mammary gland which modifies the product specificity of fatty acid synthetase. J Biol Chem 253(5): 1393-1401

22. Magee AI, Gutierrez L, Marshall CJ, Hancock JF (1989) Targeting of oncoproteins to membranes by fatty acylation. J Cell Sci Suppl 11: $149-160$

23. Mashhedi H, Blouin MJ, Zakikhani M, David S, Zhao Y, Bazile M, Birman E et al (2011) Metformin abolishes increased tumor (18)F-2fluoro-2-deoxy-D-glucose uptake associated with a high energy diet. Cell Cycle 10(16):2770-2778

24. McCleland ML, Adler AS, Shang Y, Hunsaker T, Truong T, Peterson D, Torres E et al (2012) An integrated genomic screen identifies LDHB as an essential gene for triple-negative breast cancer. Cancer Res 72(22):5812-5823. doi:10.1158/0008-5472.CAN-12-1098

25. Menendez JA, Oliveras-Ferraros C, Cufi S, Corominas-Faja B, Joven J, Martin-Castillo B, Vazquez-Martin A (2012) Metformin is synthetically lethal with glucose withdrawal in cancer cells. Cell Cycle 11(15):2782-2792. doi:10.4161/cc.20948

26. Oburoglu L, Tardito S, Fritz V, de Barros SC, Merida P, Craveiro M, Mamede J et al (2014) Glucose and glutamine metabolism regulate human hematopoietic stem cell lineage specification. Cell Stem Cell. doi:10.1016/j.stem.2014.06.002

27. Ookhtens M, Kannan R, Lyon I, Baker N (1984) Liver and adipose tissue contributions to newly formed fatty acids in an ascites tumor. Am J Physiol Regul Integr Comp Physiol 247: R146-R153

28. Panopoulos AD, Yanes O, Ruiz S, Kida YS, Diep D, Tautenhahn R, Herrerias A et al (2012) The metabolome of induced pluripotent stem cells reveals metabolic changes occurring in somatic cell reprogramming. Cell Res 22(1):168-177. doi:10.1038/cr.2011.177

29. Rafalski VA, Mancini E, Brunet A (2012) Energy metabolism and energy-sensing pathways in mammalian embryonic and adult stem cell fate. J Cell Sci 125(Pt 23):5597-5608. doi: $10.1242 /$ jcs. 114827

30. Resh MD (1996) Regulation of cellular signalling by fatty acid acylation and prenylation of signal transduction proteins. Cell Signal 8(6):403-412

31. Resh MD (1999) Fatty acylation of proteins: new insights into membrane targeting of myristoylated and palmitoylated proteins. Biochim Biophys Acta 1451(1):1-16

32. Rudolph MC, Wellberg EA, Lewis AS, Terrell KL, Merz AL, Maluf NK, Serkova NJ, Anderson SM (2014) Thyroid hormone responsive protein spot14 enhances catalysis of fatty acid synthase in lactating mammary epithelium. J Lipid Res. doi:10.1194/jlr.M044487

33. Sabine JR, Abraham S, Chaikoff IL (1967) Control of lipid metabolism in hepatomas: insensitivity of rate of fatty acid and cholesterol synthesis by mouse hepatome BW7756 to fasting and to feedback control. Cancer Res 27:793-799

34. Schlaepfer IR, Hitz CA, Gijon MA, Bergman BC, Eckel RH, Jacobsen BM (2012) Progestin modulates the lipid profile and sensitivity of breast cancer cells to docetaxel. Mol Cell Endocrinol 363(1-2):111-121. doi:10.1016/j.mce.2012.08.005

35. Sciacovelli M, Gaude E, Hilvo M, Frezza C (2014) The metabolic alterations of cancer cells. Methods Enzymol 542:1-23. doi:10.1016/ B978-0-12-416618-9.00001-7 
36. Stumvoll M, Nurjhan N, Perriello G, Dailey G, Gerich JE (1995) Metabolic effects of metformin in non-insulin-dependent diabetes mellitus. N Engl J Med 333(9):550-554. doi:10.1056/ NEJM199508313330903

37. Van de Sande T, De Schrijver E, Heyns W, Verhoeven G, Swinnen JV (2002) Role of the phosphatidylinositol 3'-kinase/PTEN/Akt kinase pathway in the overexpression of fatty acid synthase in LNCaP prostate cancer cells. Cancer Res 62(3):642-646

38. Vlashi E, Lagadec C, Vergnes L, Reue K, Frohnen P, Chan M, Alhiyari Y, Dratver MB, Pajonk F (2014) Metabolic differences in breast cancer stem cells and differentiated progeny. Breast Cancer Res Treat. doi:10.1007/s10549-014-3051-2

39. Wahdan-Alaswad R, Fan Z, Edgerton SM, Liu B, Deng XS, Arnadottir SS, Richer JK, Anderson SM, Thor AD (2013)
Glucose promotes breast cancer aggression and reduces metformin efficacy. Cell Cycle 12(24):3759-3769. doi:10.4161/cc. 26641

40. Warburg O (1956) On the origin of cancer cells. Science 123(3191): 309-314

41. Yanes O, Clark J, Wong DM, Patti GJ, Sanchez-Ruiz A, Benton HP, Trauger SA, Desponts C, Ding S, Siuzdak G (2010) Metabolic oxidation regulates embryonic stem cell differentiation. Nat Chem Biol 6(6):411-417. doi:10.1038/ nchembio. 364

42. Yang YA, Han WF, Morin PJ, Chrest FJ, Pizer ES (2002) Activation of fatty acid synthesis during neoplastic transformation: role of mitogen-activated protein kinase and phosphatidylinositol 3-kinase. Exp Cell Res 279(1):80-90 